\title{
A Cross Layer Framework for WLANs: Joint Radio Propagation and MAC Protocol
}

\author{
Nurul I. Sarkar \\ School of Computing and Mathematical Sciences, Auckland University of Technology, Auckland, New Zealand \\ nurul.sarkar@aut.ac.nz
}

\begin{abstract}
This paper proposes a cross-layer design (CLD) framework called channel-aware buffer unit multiple access (C-BUMA) for improving wireless local area network (WLAN) performance. In the framework, the radio propagation (i.e. PHY layer) is combined with the medium access control (MAC) protocol for packet transmissions. By sharing channel information with the MAC protocol, the approach reduced unnecessary packet transmissions and hence improved system performance. Through performance evaluation, we demonstrate that our CLD can significantly improve network throughput and packet delay. The proposed C-BUMA is simple and can easily be implemented in 802.11 networks without changing hardware infrastructure and no additional costs. In this paper we describe C-BUMA and present two algorithms for the implementation of the framework.
\end{abstract}

Keywords: C-BUMA, Cross-layer design, 802.11 networks.

\section{INTRODUCTION}

Research on cross-layer design (CLD) optimization has generated a lot of interest in recent years [1-4]. It shares information between protocol layers [5]. This paper proposes a CLD framework for improving the performance of IEEE 802.11 ("802.11") WLANs. The framework is based on a cross layer MAC protocol called the channel-aware buffer unit multiple access (CBUMA). The idea is to determine the status of the wireless channel and to share this knowledge with the MAC protocol. C-BUMA provides better system performance because it delivers more packets when channel status is good and pauses transmission when signal is "very weak". The proposed framework is simple and can easily be implemented in 802.11 networks requiring no hardware changes and no additional costs.

The CLD has been considered by the IEEE Mobile Broadband Wireless Access Networks Group with the goal of improving system performance in both uplink and downlink [6].

The remainder of this paper is structured as follows. Section II reviews literature on CLD for WLANs. Section III describes the proposed CLD framework. The CLD algorithms are also presented in this section. The performance of the proposed CLD is evaluated in Section IV. Section V discusses system implications and Section VI concludes the paper.

\section{LITERATURE REVIEW}

To improve network performance, the CLD framework has been proposed by many network researchers. This section reports only a selected set of literature that is indicative of the range of approaches used for CLD optimization to improve WLAN performance.

Pham et al. [7] proposed a method for predictability of Rayleigh fading channels to improve the performance of wireless networks. The idea is to share channel status information (CSI) with the upper protocol layers. Having CSI before packet transmissions, the upper layers know whether the channel is good enough to guarantee a successful transmission. This method was shown to improve network throughput.

Ge et al. [8] proposed a CLD method for efficient multicast communications where the transport layer erasure coding is combined with the MAC layer. Data rate optimization for single-input-single-output (SISO) and multiple-input-multiple-output (MIMO) links are investigated.

Yuan et al. [9] proposed a CLD called opportunistic cooperative MAC (OC-MAC). It determines the best relay station between source and destination based on instantaneous channel measurements. OC-MAC uses relay stations for data transmission to improve system performance.

Khan et al. [2] proposed a rate adaptation CLD framework for 802.11 networks. In Khan's solution, the data rate is adapted to the changing channel condition, application preferences and MAC timing constraints. It is based on a cross layer approach involving application layer with rate adaptation.

Lee and Chung [10] proposed a CLD for video streaming over wireless networks. It is based on the joint optimization of rate adaptation and application quality adaptation which is basically the combination of two earlier proposals: rate adaptation [2] and video quality [11]. The rate adaptation method adjusts the transmission rate at the transmitting-antenna and informs the upper layer about the rate limits. The quality adaptation method then utilizes this rate information to adjust the quality of the video streams.

Choudhury and Gibson [12] proposed a CLD method to optimize single user throughput using transmitted bit rate and payload length as a function of channel state for fading channels. The CLD method jointly optimizes payload length and data rate for a given channel state.

To overcome the unfairness problems in 802.11 networks, Dunn et al. [13] proposed a CLD method to provide "rate proportional fairness" to the 802.11 
WLANs. The key idea is to adjust the packet length so that low bit rate stations can send less bytes per packet and high bit rate stations send more data.

The CLD approaches reviewed are grouped into five main categories shown in Table I. The proposed CLD described in Section III uses the idea of sharing wireless channel with an access protocol for optimum transmissions. It is a joint radio propagation and MAC method drawing ideas from Rayleigh channel predictability [7]. The channel-aware MAC protocol (CBUMA) is the key element of the proposed framework.

TABLE I. CATEGORIES OF CLD APPROACHES REVIEWED

\begin{tabular}{|l|l|}
\hline Cross-layer & Example of CLD proposals/approaches \\
\hline PHY-MAC & $\begin{array}{l}\text { Rayleigh channel predictability [7] } \\
\text { Rate adaptation and payload length [12] } \\
\text { Opportunistic cooperative MAC [9] }\end{array}$ \\
\hline $\begin{array}{l}\text { PHY-Data link- } \\
\text { Application }\end{array}$ & Joint quality and rate adaptation [10] \\
\hline PHY-Application & Rate adaptation solution [2] \\
\hline $\begin{array}{l}\text { PHY-Data link- } \\
\text { IP }\end{array}$ & Rate proportional 802.11 fairness [13] \\
\hline MAC-Transport & Rate optimization and transport layer [8] \\
\hline
\end{tabular}

\section{THE PROPOSED CLD METHOD}

Figure 1 shows a block diagram of the proposed CLD framework, differs from the earlier work described in Section II. In the framework, radio propagation modeling and MAC protocol are integrated into one single layer. The propagation modeling predicts the wireless channel state and shares the CSI with the MAC protocol. Having access to the CSI before transmitting a packet, the MAC protocol can estimate whether the channel is "good" enough to guarantee a successful transmission.
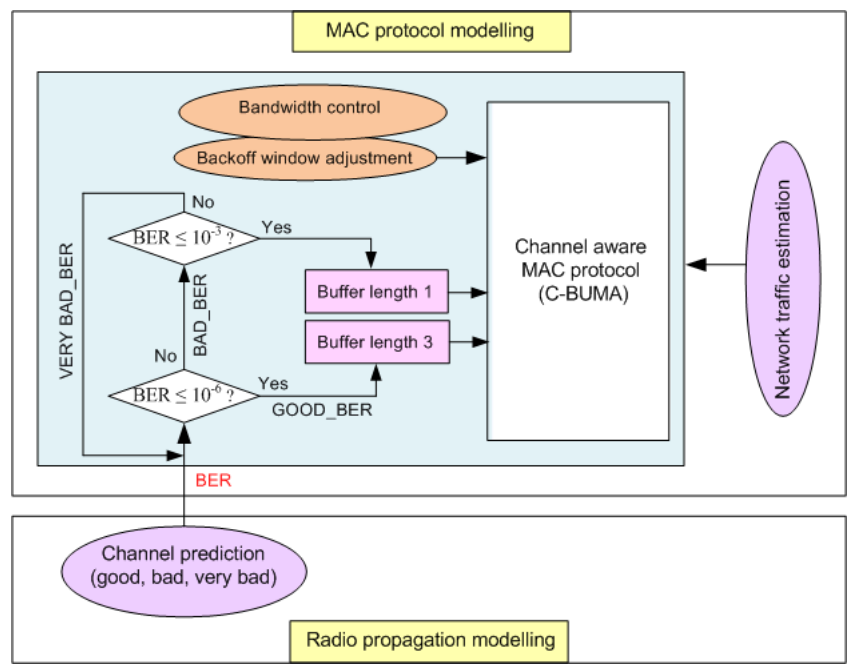

Figure 1. The proposed CLD framework for WLANs.

The receiving station can easily determine the channel status by examining the received signal's bit error rate (BER). The channel's BER can be indicated in DCF by setting a special flag in the packet trailer. However, to investigate the impact of BER on WLAN performance, it is important to classify the wireless channel state based on BER. Table II lists the definition of the three channel states: good, bad, and very bad.
TABLE II. THREE STATES OF A CHANNEL

\begin{tabular}{|c|l|}
\hline $\begin{array}{c}\text { Channel } \\
\text { status }\end{array}$ & \multicolumn{1}{|c|}{ Definition } \\
\hline good & $\begin{array}{l}\text { The wireless link is relatively "clean" and is } \\
\text { characterized by a very small BER, which is denoted } \\
\text { by GOOD_BER. }\end{array}$ \\
\hline bad & $\begin{array}{l}\text { The wireless link is in a condition characterized by } \\
\text { increased BER (in the order of } 10^{-6} \text { to } 10^{-3} \text { ), which is } \\
\text { denoted by BAD_BER [14]. }\end{array}$ \\
\hline very bad & $\begin{array}{l}\text { The BER is greater than } 10^{-3}, \text { denoted by } \\
\text { VERYBAD_BER. }\end{array}$ \\
\hline
\end{tabular}

C-BUMA transmits a packet based on CSI. If the channel state is bad (i.e. BAD_BER), C-BUMA attempts to transmit a packet but limits the packet scheduling to 1 packet by setting the buffer unit length to one. For a GOOD_BER, C-BUMA properly utilizes the channel by transmitting a larger packet containing one or more MAC Protocol Data Unit (MPDU) that appears as a MAC Segment Data Unit (MSDU) in the MAC layer with a single header and a trailer. This strategy significantly improves network throughput because it requires less transmission overhead than DCF to send the same payload.

More details about the packet scheduling strategy of C-BUMA, including optimization of the buffer unit length can be found in [15]. However, in the case of a VERYBAD_BER, C-BUMA pauses packet transmission as it has a very low probability of being received correctly by the receiving station. This strategy saves the network from wasting both transmitter power and channel bandwidth and hence the overall network performance is improved. The channel prediction and transmission control algorithms are described next.

\section{A. Channel Prediction Algorithm}

Figure 2 outlines the channel prediction algorithm. This algorithm estimates the channel state based on received signal strength (RSS) values. Under slow Rayleighfading the duration of the channel maintaining a "good" state is generally longer than the packet transmission time.

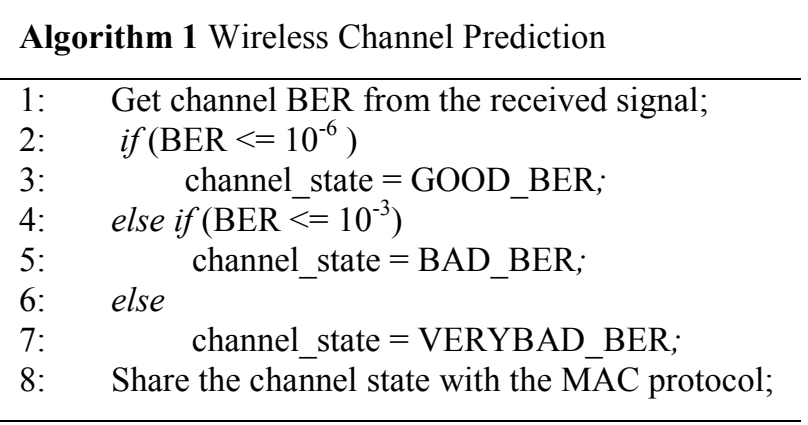

Figure 2. Wireless channel prediction algorithm.

Using the prediction, the receiving station can determine whether the packet will be received correctly in the next transmission round. The proposed channel prediction algorithm is simple and does not require 
extensive computation. Therefore, it is easy to implement in real systems.

\section{B. Transmission Control Algorithm}

The 802.11 WLANs are slotted where a station is allowed to transmit a packet at the beginning of an empty slot. A slot can be either 'busy' or 'empty'. A single bit (B) in the header represents slot status. An empty slot has $\mathrm{B}=0$ and a slot carrying a packet has $\mathrm{B}$ $=1$. Table III defines the two types of slot.

TABLE III. BUSY AND EMPTY SLOT.

\begin{tabular}{|l|l|}
\hline Slot status & Definition \\
\hline busy & A slot is occupied carrying a packet. \\
\hline empty & $\begin{array}{l}\text { A slot does not carry a packet at present, can be } \\
\text { used for future transmissions. }\end{array}$ \\
\hline
\end{tabular}

Figure 3 outlines the transmission control algorithm to be executed at each active station on the network. The proposed C-BUMA schedules a packet for transmission based on the knowledge of CSI obtained from the received packet. C-BUMA improves the network performance further by scheduling three packets (optimum buffer length) for stations that gain network access in good channel states.

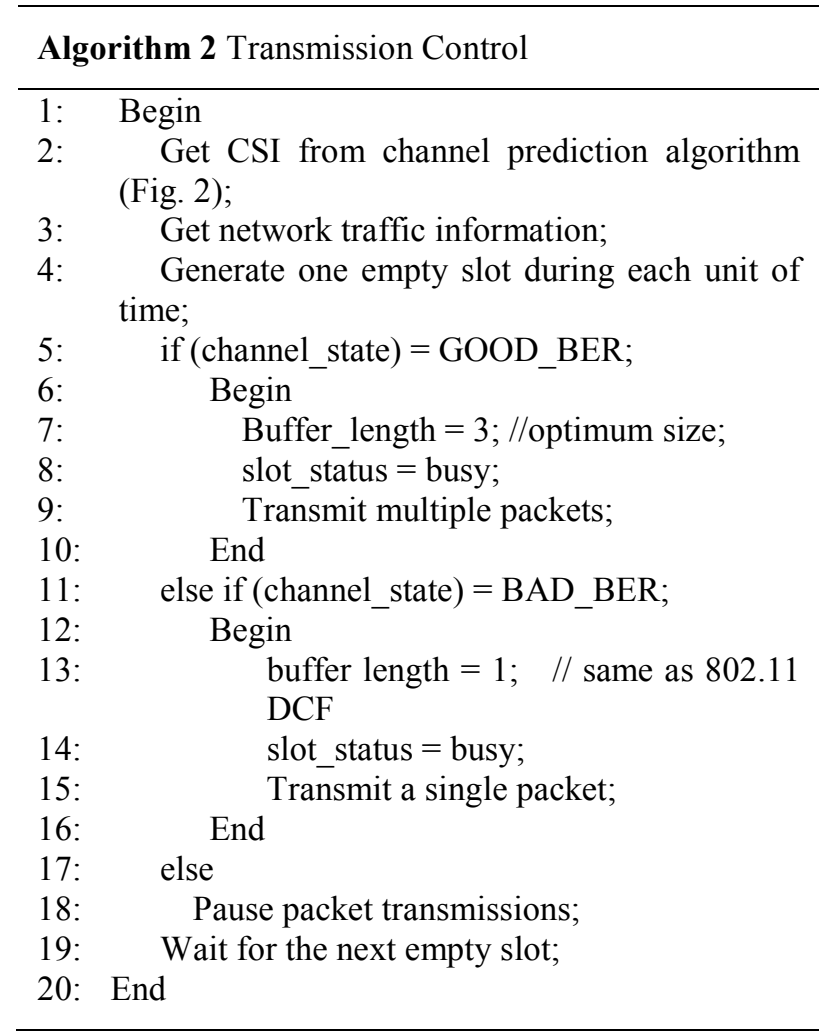

Figure 3. Transmission control algorithm executed at each active station.

When the channel state is bad (BAD_BER), the CBUMA transmits a single packet by setting the buffer unit length to one. When the channel is in a fade (VERYBAD_BER), source/destination suspends the transmission for an average fade duration which depends on the Doppler frequency and the RMS value of the received power [7]. The notification of the incoming fade can be implemented in DCF by setting a special flag in the header of the clear-to-send (CTS) packet or the ACK packet. Upon hearing this CTS the neighbouring stations set their network allocation vectors (NAVs) to the fade duration. The channel can then be released for other transmissions.

\section{Performance Evaluation}

\section{A. Performance Metrics and Simulation Parameters}

The performance of the proposed CLD framework is evaluated by ns-2.31 simulations. The performances of 802.11 DCF ("DCF") with and without the proposed CLD are compared. The CLD was evaluated by measuring network mean throughput and packet delay. The throughput (Mbps) is the mean rate of successful message delivery over a communication channel. The mean packet delay is defined as the average time (seconds) from the moment the packet is generated until the packet is fully despatched from that station.

A wireless ad hoc network with $\mathrm{N}=10$ stations, Pareto packet arrivals, data packet length of 1500 bytes, UDP streams, offered loads from $10 \%$ to $100 \%$, and shadowing propagation model with $\sigma=7 \mathrm{~dB}$ are used in the simulations [16]. All sources and receivers have an omnidirectional antenna of height $1.5 \mathrm{~m}$. Hidden and exposed station problems are not considered. Table IV lists simulation parameters. All simulation results report the steady state behavior of the network and were obtained with a relative statistical error $\leq 1 \%$ at $99 \%$ confidence level. Each simulation is run for 10 minutes simulated time to obtain steady state results.

\section{TABLE IV. SIMULATION PARAMETERS.}

\begin{tabular}{|c|c|}
\hline Parameter & Value \\
\hline Data rate & $11 \mathrm{Mbps}$ \\
\hline Basic rate & $2 \mathrm{Mbps}$ \\
\hline Wireless cards & $802.11 \mathrm{~b}$ \\
\hline Slot duration & $20 \mu \mathrm{s}$ \\
\hline SIFS & $10 \mu \mathrm{s}$ \\
\hline DIFS & $50 \mu \mathrm{s}$ \\
\hline MAC header & 30 bytes \\
\hline CRC & 4 bytes \\
\hline PHY header & $96 \mu \mathrm{s}$ \\
\hline Packet/Traffic type & UDP \\
\hline Packet arrivals & Pareto \\
\hline RTS-CTS & Off \\
\hline PHY modulation & DSSS \\
\hline Propagation model & Shadowing \\
\hline CWmin & 31 \\
\hline CWmax & 1023 \\
\hline Simulation time & 10 minutes \\
\hline
\end{tabular}

\section{$B$. Results and Discussions}

The impact of CLD on network mean throughput for UDP traffic is illustrated in Fig. 4. The network mean throughput increases with traffic load and becomes saturated at $90 \%$ loads. The CLD provides higher throughput than the network without CLD, especially under medium-to-high traffic loads. For example, using CLD in an ad hoc network with $\mathrm{N}=10$ stations at $60 \%$ loads, the throughput can be increased by approximately 
$12 \%$. The main conclusion is that the CLD improves network throughput significantly in an obstructed office environment for UDP under medium-to-high loads.

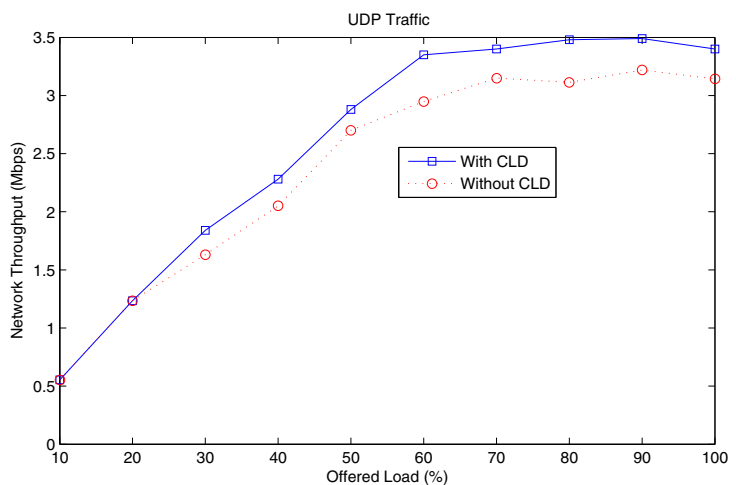

Figure 4. Network mean throughput with and without CLD for UDP traffic.

The impact of CLD on network mean packet delay for UDP traffic is illustrated in Fig. 5. The packet delay increases with traffic load and becomes saturated at $90 \%$ loads. The CLD approach improves network mean packet delays by 7 to $56 \%$. The main conclusion is that stations using CLD have a substantially lower mean packet delay than stations without CLD especially under medium-to-high loads.

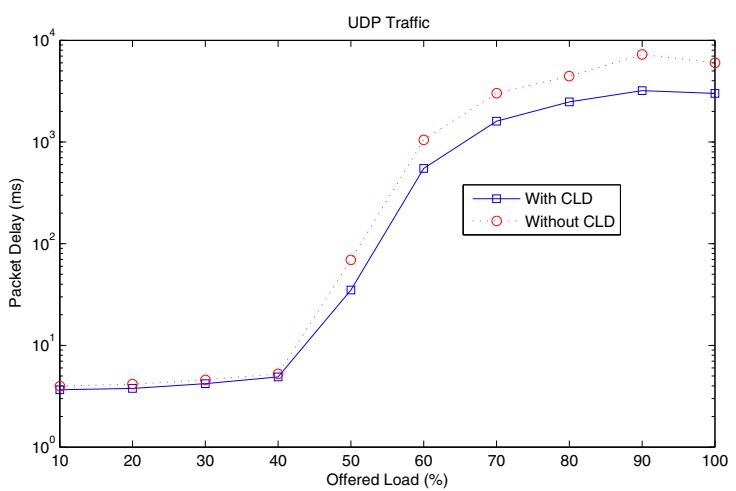

Figure 5. Network mean packet delay with and without CLD for UDP traffic.

Table $\mathrm{V}$ compares the performance of the proposed CLD with another CLD protocol proposed by Pham et al. [7] which we called Pham's CLD. For the proposed CLD, the empirical results are obtained from simulation runs with the controlled final precision of steady-state estimates. For Pham's CLD, we use numerical results from Pham's paper [7]. Unfortunately, we had numerical results for network throughput and packet drops only with identical network configuration as the proposed CLD. However, the proposed CLD provides higher network throughput than that of Pham's CLD. The main conclusion is that when the proposed CLD is used in place of Pham's CLD for a 10-station network, one can achieve significantly higher throughput and lower packet dropping, especially under high traffic loads.
TABLE V. COMPARISON OF THE PROPOSED CLD AND PHAM'S CLD.

\begin{tabular}{|l|c|c|}
\hline Performance metrics & Proposed CLD & Pham etal. CLD [7] \\
\hline $\begin{array}{l}\text { Throughput improvement } \\
\text { using CLD (TCP traffic) }\end{array}$ & $40 \%$ & $5 \%$ \\
\hline $\begin{array}{l}\text { Packets dropped due to } \\
\text { low power (UDP traffic) }\end{array}$ & 81064 packets & 1000000 packets \\
\hline $\begin{array}{l}\text { Network configuration (proposed CLD and Pham's CLD): } \\
\text { Ad hoc network with 10 active nodes; packet length of 512 bytes; } \\
\text { RTS/CTS ON; high traffic loads; AODV routing protocol. }\end{array}$ \\
\hline
\end{tabular}

The ns-2 simulation model was validated through real measurements using $802.11 \mathrm{~b}$ wireless cards [17]. In addition, ns-2 results were compared with the results obtained from OPNET Modeler [18] and a good match between two sets of results further validated the simulation models.

\section{BENEFITS AND SYSTEM IMPLICATIONS}

The wireless channel state varies over time and space, and the received signal can go into deep fades [16]. If the proposed CLD is not used the MAC layer is not notified about the wireless channel status. Therefore, Transmitter (Tx) keeps sending packets that are discarded as a result of weak RSS values at Receiver (Rx). Therefore, using the proposed CLD approach, one can obtain the following improvements. First, it prevents the sender from unnecessary transmissions, which leads to the reduction of power consumption for transmission. Second, it saves transmission bandwidth that can be used for transmitting payload and hence higher network throughput can be achieved. The channel prediction and the transmission control algorithms outlined in Figs. 2 and 3, respectively improve considerably the network performance as is evident from the simulation results presented in Section IV. The proposed CLD algorithms are straightforward and can be implemented easily without changing any existing DCF hardware.

\section{CONCLUSIONS}

This paper proposed a CLD framework for improving the performance of 802.11 WLANs. The framework is based on C-BUMA, a channel aware MAC protocol. The proposed CLD combines the radio propagation and the MAC layer into one layer. By sharing channel information with the MAC protocol, the approach reduced unnecessary packet transmissions, and therefore significantly improved system performance. Simulation results have shown that the network achieved up to $13.5 \%$ higher throughput and 56\% lower packet delay with CLD.

For the implementation of the proposed CLD framework, channel prediction and transmission control algorithms were presented. These algorithms are simple and easy to implement in 802.11 WLANs requiring no hardware changes. A future paper will report a robust rate adaptive QoS aware MAC protocol for multimedia WLANs. 


\section{REFERENCES}

[1] V. Naware and L. Tong, "Cross layer design for multiaccess communication over Rayleigh fading channels," IEEE Transactions on Wireless Communications, vol. 7, no. 3, pp. 1095-1103, 2008.

[2] S. Khan, S. A. Mahmud, K. K. Loo, and H. S. Al-Raweshidy, "A cross layer rate adaptation solution for IEEE 802.11 networks," Computer Communications, vol. 31, no. 8, pp. 1638-1652, 2008.

[3] M. Lee, M. Kang, M. Kim, and J. Mo, "A cross-layer approach for TCP optimization over wireless and mobile networks," Computer Communications, vol. 31, no. 11, pp. 2669-2675, 2008.

[4] Z. Qian and Z. Ya-Qin, "Cross-layer design for QoS support in multihop wireless networks," Proceedings of the IEEE, vol. 96, no. 1, pp. 64-76, 2008.

[5] The OSI Reference Model. Retrieved February 2, 2010, from http://www.thecertificationhub.com/networkplus/the_osi_ref_mo del.htm

[6] S. Shakkottai, T. S. Rappaport, and P. C. Karlsson, "Cross-Layer Design for Wireless Networks," IEEE Communications Magazine, vol. 41, no. 10, pp. 74-80, 2003.

[7] P. P. Pham, S. Perreau, and A. Jayasuriya, "New cross-layer design approach to ad hoc networks under Rayleigh fading," IEEE Journal on Selected Areas in Communications, vol. 23, no. 1, pp. 28-39, 2005.

[8] W. Ge, J. Zhang, and S. Shen, "A cross-layer design approach to multicast in wireless networks," IEEE Transactions on Wireless Communications, vol. 6, no. 3, pp. 1063-1071, 2007.

[9] Y. Yuan, Z. Baoyu, L. Wentao, and C. D., "An opportunistic cooperative MAC protocol based on cross-layer design," presented at the 2007 International Symposium on Intelligent Signal Processing and Communication Systems (ISPACS '07), November 28 - December 01, 2007, pp. 714-717.

[10] S. Lee and K. Chung, "Joint quality and rate adaptation scheme for wireless video streaming," presented at the 22nd International
Conference on Advanced Information Networking and Applications (AINA '08), March 25-28, 2008, pp. 311-318.

[11] P. Cheng, Z. Zhang, H.-H. Chen, and P. Qiu, "A framework of cross-layer design for multiple video streams in wireless mesh networks," Computer Communications, vol. 31, no. 8, pp. 1529 1539, 2008.

[12] S. Choudhury and J. D. Gibson, "Payload length and rate adaptation for multimedia communications in wireless LANs," IEEE Journal on Selected Areas in Communications, vol. 25, no. 4, pp. 796-807, 2007.

[13] J. Dunn, M. Neufeld, D. Sheth, D. Grunwald, and J. Bennett, "A practical cross-layer mechanism for fairnes in 802.11 networks," Mobile Networks and Applications, vol. 11, no. 1, pp. 37-45, 2006.

[14] P. Nicopolitidis, G. I. Papadimitriou, M. S. Obaidat, and A. S. Pomportsis, "TRAP: a high performance protocol for wireless local area networks," Computer Communications, vol. 25, no. 11, pp. 1058-1065, 2002.

[15] N. I. Sarkar and K. W. Sowerby, "Buffer unit multiple access (BUMA) protocol: an enhancement to IEEE 802.11b DCF," presented at the IEEE Global Telecommunications Conference (GLOBECOM '05), St. Louis, USA, November 28 - December 2, 2005, pp. 2584-2588.

[16] T. S. Rappaport, Wireless communications: principles and practice, 2nd ed. Englewood Cliffs, NJ: Prentice-Hall, 2002.

[17] N. I. Sarkar and K. W. Sowerby, "Wi-Fi performance measurements in the crowded office environment: a case study," presented at the 10th IEEE International Conference on Communication Technology (ICCT ' 06), Guilin, China, November 27-30, 2006, pp. 37-40.

[18] OPNET Modeler. Retrieved June 20, 2010, from www.opnet.com 